Strahlenther Onkol 2013 · 189:1056-1057

DOI 10.1007/s00066-013-0475-2

Online publiziert: 27. Oktober 2013

(c) Springer-Verlag Berlin Heidelberg 2013
A. Dal Pra · D.M. Aebersold

Universitätsklinik für Radio-Onkologie, Inselspital, Universität Bern

\section{Radiotherapie mit rechtwinkligen Feldern im Vergleich zu konformalen Feldern}

\author{
Weniger Therapieversager bei \\ Hochrisiko-Prostatakarzinompatienten
}

\section{Originalbeitrag}

Heemsbergen WD, Al-Mamgani A, Witte MG et al (2013) Radiotherapy with rectangular fields is associated with fewer clinical failures than conformal fields in the high-risk prostate cancer subgroup: results from a randomized trial. Radiother Oncol 107:134-139

Hintergrund. Patienten mit einem Hochrisiko-Prostatakarzinom haben außerhalb der Prostata ein erhöhtes Risiko für subklinische Tumormanifestationen inklusive einer Mikrometastasierung. Mit den heute angewendeten Bestrahlungstechniken wird aufgrund engerer Sicherheitssäume die periprostatisch applizierte Dosis reduziert. Die Autoren [1] untersuchten, ob die Verwendung von rechtwinkligen Feldern mit weniger Therapieversagen assoziiert ist im Vergleich zu konformalen Feldern mit einer geringeren periprostatischen Dosis.

Patienten und Methoden. Es wurden 164 Hochrisiko-Patienten aus einer Studienpopulation von 266 Patienten mit den Tumorstadien T1-4 N0 M0 ausgewählt, welche randomisiert in einem Arm eine „rechtwinklige“ Bestrahlung ( $\mathrm{n}=79)$ und im anderen Arm eine „konformale“ Bestrahlung erhielten. Die vorgeschriebene Dosis betrug 66 Gy auf die Prostata und die Samenblasen inklusive eines $15 \mathrm{~mm}$ breiten Sicherheitssaums. Die Zahl der Therapieversager (in- und exklusive lokales Therapieversagen) wurde verglichen. Die periprostatische Dosis wurde mittels einer „Inter-Patient-Mapping“-Methode berechnet.

Ergebnisse. Das mediane Follow-up betrug 34 Monate. Im Arm "rechtwinklig“ traten 9 Therapieversager auf im Vergleich zu 24 im Arm „konformal“ ( $p=0,012)$. Die Zahl der Therapieversager außerhalb der Prostata betrug 7 respektive $19(\mathrm{p}=0,025)$. Es wurden Dosisdifferenzen von 5-35 Gy zwischen den beiden Armen in den periprostatischen Regionen festgestellt.

Schlussfolgerungen der Autoren. Das Risiko für eine frühe Tumorprogression ist signifikant geringer bei Patienten, welche mit rechtwinkligen Feldern bestrahlt werden. Ein Therapieversagen kann möglicherweise dadurch vermindert werden, dass man Regionen mit einem vermuteten subklinischen Tumorbefall außerhalb der Prostata mitbestrahlt.

\section{Kommentar}

Die Autoren adressieren in dieser Studie das Thema des optimalen Bestrahlungsvolumens bei Patienten mit einem Hochrisiko-Prostatakarzinom. Aus der anatomischen Perspektive sind dabei zwei Erklärungen der Resultate möglich: Effekte durch die Bestrahlung des periprostatischen Gewebes und die Rolle der prophylaktischen Lymphknotenbestrahlung.

Die image-guided Radiotherapie (IGRT) erlaubt durch den Einsatz der Bildgebung am Linearbeschleuniger die Erfassung und Korrektur von Positionsabwei- chungen der Prostata und damit die Verkleinerung von Sicherheitssäumen für die PTV-Definition. Dabei werden üblicherweise ausschließlich Positionskorrekturen an der Prostataposition sowie der Samenblasenbasen vorgenommen, ungeachtet möglicher subklinischer Tumormanifestationen periprostatisch. Neben der Gefahr der inadäquaten Reduktion der PTV-Säume aus Sicht Prostataabdeckung kommt entsprechend die Gefahr einer Untertherapie subklinischer periprostatischer Tumorausläufer dazu. Tatsächlich haben bereits einige Studien auf die Gefahren einer zu knappen PTV-Saum-Definition hingewiesen, beispielsweise im Kontext von Patienten mit extendiertem Rektum. So beschrieben z. B. Engels et al. [2] eine erhöhte Rate an biochemischem Versagen bei Patienten, welche eine markerbasierte IGRT erhielten, im Vergleich zu Patienten, deren Positionierung aufgrund der knöchernen Strukturen erfolgte.

Der in der hier kommentierten Studie berichtete negative Effekt einer konformalen Bestrahlung könnte auch durch die verminderte Dosisbelastung von okkulten Lymphknotenmetastasen bei diesem Hochrisiko-Patientenkollektiv erklärt werden. Das Risiko einer Fernmetastasierung ist ja tatsächlich wesentlich auch vom Auftreten von Lymphknotenmetastasen abhängig. Nichtsdestotrotz bleibt die Bedeutung der elektiven periprostatischen respektive pelvinen Lymphknotenbestrahlung umstritten [3]. Obwohl in vielen Zentren routinemässig eingesetzt, konnte bislang keine prospektiv-randomisierte Stu- 
die die Vorteile einer prophylaktischen Beckenbestrahlung zweifelsfrei zeigen. Die laufende Phase-III-Studie 0924 der RTOG wird hoffentlich in dieser Frage eine Antwort bringen.

Heutzutage gilt für bestrahlte Patienten mit einem Hochrisiko-Prostatakarzinom eine Radiotherapie, kombiniert mit einer Hormontherapie, als Standard [4]. In der aktuellen Studie hatten aber nur 16\% eine Hormontherapie bekommen. Es darf wohl davon ausgegangen werden, dass die beobachteten Unterschiede der Metastasierung zwischen den beiden Armen nicht aufgetreten wären, hätten die Patienten eine nach heutigem Standard ausgerichtete Kombinationstherapie erhalten - jedenfalls nicht innerhalb von nur 34 Monaten.

So interessant die von den Autoren adressierten Fragen auch sind, so schwach sind die Füße, auf denen die Antworten stehen. Der relevante Unterschied im Outcome zeigte sich nur hinsichtlich des Auftretens von Fernmetastasen, nicht aber bezüglich von lokoregionärem Therapieversagen. Wenn Effekte unterschiedlicher Bestrahlungsvolumina Thema sind, würde man sie ja in erster Linie beim lokoregionären Geschehen erwarten. Übrigens hatte die Studie auch nicht die statistische Power, um die Haupthypothese mit genügend $\mathrm{Pa}$ tientenzahlen und Ereignissen zu belegen. Kommt hinzu, dass in Abwesenheit einer Stratifikation für bekannte Risikofaktoren relevante Ungleichgewichte in der Patientenverteilung auszumachen waren - Ungleichgewichte, die wohl alleine die berichteten Unterschiede zwischen den beiden Therapiearmen zu erklären vermögen. Zudem erfahren wir in der Studie nichts über das krankheitsspezifische Überleben oder gar das Gesamtüberleben beider Behandlungsgruppen.

\section{Fazit}

Was bleibt, ist ein Papier, welches zur Hypothesengenerierung dienen mag. Antworten auf die Frage der Bedeutung einer periprostatischen bzw. lymphatischen Bestrahlung werden nur mit prospektiv randomisierten Studien zu generieren sein, die diese Frage als primären Endpunkt untersuchen.

Alan Dal Pra und Daniel M. Aebersold, Bern

\section{Korrespondenzadresse}

Prof. Dr. med. D.M. Aebersold

Universitätsklinik für Radio-Onkologie,

Inselspital, Universität Bern

Bern

Schweiz

daniel.aebersold@insel.ch

\section{Einhaltung ethischer Richtlinien}

Interessenkonflikt. A. Dal Pra und D.M. Aebersold geben an, dass kein Interessenkonflikt besteht.

\section{Literatur}

1. Heemsbergen WD, Al-Mamgani A, Witte MG et al (2013) Radiotherapy with rectangular fields is associated with fewer clinical failures than conformal fields in the high-risk prostate cancer subgroup: results from a randomized trial. Radiother Oncol 107:134-139

2. Engels B, Soete G, Verellen D et al (2009) Conformal arc radiotherapy for prostate cancer: increased biochemical failure in patients with distended rectum on the planning computed tomogram despite image guidance by implanted markers. Int J Radiat Oncol Biol Phys 74:388-391

3. Morikawa LK, Roach M 3rd (2011) Pelvic nodal radiotherapy in patients with unfavorable intermediate and high-risk prostate cancer: evidence, rationale, and future directions. Int J Radiat Oncol Biol Phys 80:6-16

4. Dal Pra A, Cury FL, Souhami L (2010) Combining radiation therapy and androgen deprivation for localized prostate cancer-a critical review. Curr Oncol $17: 28-38$ 